\title{
ORIGINAL
}

\section{TENDENCIA Y DISTRIBUCIÓN MUNICIPAL DE LA INCIDENCIA DE CÁNCER DE MAMA EN EL ÁREA DE SALUD DE LEÓN (1996-2010) (*)}

Lidia García Martínez (1), Marina Pollán Santamaría (2,3), Gonzalo López-Abente (2,3), María Mercedes Sánchez Jacob (4), Andrés García Palomo (5), Raquel González Martínez (6), Emiliano Honrado Franco (6) y Vicente Martín Sánchez (1,3).

(1) Grupo de Investigación en Interacción Gen-Ambiente-Salud. Universidad de León.

(2) Centro Nacional de Epidemiología, Instituto de Salud Carlos III, Madrid.

(3) Centro de Investigación Biomédica en Red de Epidemiología y Salud Pública (CIBERESP).

(4) Dirección General de Salud Pública. Junta de Castilla y León.

(5) Servicio de Oncología, Complejo Asistencial Universitario de León.

(6) Servicio de Anatomía Patológica, Complejo Asistencial Universitario de León.

(*) Financiado con recursos propios del grupo de investigación en Interacción Gen-Ambiente-Salud. Universidad de León.

No existen conflictos de interés.

\begin{tabular}{|c|c|}
\hline $\begin{array}{l}\text { RESUMEN } \\
\text { Fundamentos: El cáncer de mama es el más frecuente en las muje- } \\
\text { res. El objetivo del presente estudio fue analizar la incidencia y distribu- } \\
\text { ción geográfica del cáncer de mama invasivo en el área de salud de León } \\
\text { (ASL). }\end{array}$ & $\begin{array}{l}\text { ABSTRACT } \\
\text { Municipal Distribution and Trend of the } \\
\text { Incidence of Breast Cancer in the Health } \\
\text { Area of León, Spain (1996-2010) }\end{array}$ \\
\hline
\end{tabular}

Métodos: Estudio observacional descriptivo en el que se incluyeron mujeres con diagnóstico de neoplasia maligna de mama (CIE-9:174, CIE10: C50) del Registro Hospitalario de Tumores del Centro Asistencial Universitario de León, entre 1/1/1996 y 31/12/2010 y con residencia en el ASL. Para el análisis de la distribución espacial se estimaron los riesgos relativos (RR) municipales suavizados mediante el ajuste del modelo de Besag, York y Mollié y sus probabilidades posteriores de que los RR fuesen $>1$ (PP), utilizando métodos bayesianos.

Resultados: Se incluyó un total de 2.379 casos. El número de casos nuevos y las tasas de incidencia brutas en cada trienio fueron de 72,7 (1996-1998) a 101,5 (2008-2010) por 100.000 mujeres. Las tasas a población europea por 100.000 mujeres ascendieron de 58,0 en el primer trienio y a 69,4 en el último. Se observó un incremento anual promedio del 1,3\%. Varios municipios del ASL presentaron riesgos superiores al 10 $\%$. Las PP solo fueron superiores a 0,9 en el municipio de León.

Conclusiones: Las tasas observadas son de las más bajas de España Sin embargo, el número de casos y las tasas de incidencia seincrementaron de manera mantenida en el periodo estudiado.

Palabras clave: Cáncer de mama. Incidencia. Tendencias. Análisis de áreas pequeñas.

\section{Correspondencia}

Lidia García Martínez.

Universidad de León. Escuela universitaria de Ciencias de la Salud.

Área de medicina preventiva y Salud Pública.

Campus de Vegazana s/n

24071 León

987293110

Background: Breast cancer is the most common amongst women. The aim of this study was to analyze the incidence and geographical distribution of breast cancer in the health area of León.

Methods: We designed an observational descriptive study that included women enrolled in the Hospital Tumor Registry of the Centro Asistencial Universitario in León with a diagnosis of breast malignant neoplasm (ICD-9: 174, ICD-10: 50) between 01/01/1996 and 31/12/2010 and resident in the health area of León. To study the spatial distribution, we estimated municipal relative risks (RR) smoothed by fitting the Besag, York and Mollié model and the posterior probability (PP) of RR $>1$ using Bayesian methods.

Results: A total of 2379 cases were included. The number of new cases and the crude incidence rate have both increased in every triennium, from 72,7 (1996-1998) to 101,5 (2008-2010) per 100,000 women. The age adjusted rates per 100,000 women (European standard population) increased from 58,0 during the first triennium to 69,4 during the last one. An average annual increase of $1,3 \%$ was observed. Several municipalities from the health area of León showed risks higher than a $10 \%$. The PP were higher than 0.9 only in the municipality of León.

Conclusion: The observed rates are among the lowest in our country. Nevertheless, the number of cases and the incidence rates have increased progressively.

Keyword: Breast cancer. Incidence. Trends . Small area analysis. 


\section{INTRODUCCIÓN}

El cáncer de mama es el más frecuente en las mujeres. Se estima que en 2008 se produjeron en el mundo 1.383.000 casos nuevos y 458.000 muertes, suponiendo el $23 \%$ de los cánceres y el $14 \%$ de las muertes por cáncer en las mujeres. Estas cifras suponen que un $4 \%$ de las mujeres padecerán este tumor antes de los 74 años y un 1,3\% morirá a causa de él ${ }^{1}$.

Las tasas de incidencia y mortalidad difieren de unas regiones a otras y de manera general los países más desarrollados presentan tasas más elevadas que los no desarrollados ${ }^{1}$. Australia, Nueva Zelanda, Norteamérica y el norte y oeste de Europa presentan las tasas estandarizadas de incidencia más elevadas muy próximas a los 90 casos nuevos por 100.000 mujeres y año .

En Europa es también el primer cáncer en las mujeres, tanto en incidencia como en mortalidad, con unas tasas estimadas, estandarizadas según la población de la Unión Europea en 2012 (UE-27), de 109 y 22 por cada 100.000 mujeres respectivamente ${ }^{3}$. Se estima que España presenta unas tasas de incidencia y mortalidad inferiores a la media de la UE-27, con tasas ajustadas según la población europea de 85 casos nuevos y alrededor de 17 muertes por cada 100.000 mujeres y año ${ }^{3}$. Esto supone que cada año se diagnostican unos 25.000 casos nuevos y se producen unas 6.000 muertes por esta causa.

El tumor maligno de cáncer de mama presenta una gran influencia hormonal, un marcado componente genético y un papel importante de los estilos de vida (consumo de alcohol, tratamiento hormonal sustitutivo, tabaco, obesidad, inactividad física...etc) ${ }^{4}$, si bien los factores de riesgo difieren en las mujeres pre y posmenopáusicas. En las primeras los factores hereditarios son más relevantes y los tumores tienden a ser más agresivos, mientras que en las en las mujeres posmenopáusicas generalmente son más importantes la obesidad y los antecedentes reproductivos, siendo en ellas menos agresivos los tumores ${ }^{5}$.

Durante las últimas décadas, la incidencia de cáncer de mama se ha incrementado en todo el mundo dándose los mayores aumentos en los países que históricamente tenían un menor riesgo. En algunos de los países desarrollados se ha objetivado una reducción transitoria de la incidencia en mujeres posmenopáusicas, fundamentalmente debido al abandono del tratamiento hormonal sustitutivo ${ }^{5}$. En nuestro país se ha observado un incremento mantenido de la incidencia en torno a un $3 \%$ anual durante las últimas décadas en las mujeres premenopáusicas, suponiendo un tercio de los diagnósticos de cáncer de mama en España y el $16 \%$ de la mortalidad ${ }^{6,7}$.

El objetivo del presente estudio es valorar la tendencia y distribución geográfica del cáncer de mama en el área de salud de León.

\section{MATERIAL Y MÉTODOS}

Para estimar la incidencia de cáncer de mama en los municipios del área de salud se diseñó un estudio observacional y descriptivo utilizando los datos del Registro Hospitalario de Tumores del Centro Asistencial Universitario de León, el cual se guía por el Manual de procedimientos en los Registros Hospitalarios de Tumores de la Sociedad Española de Anatomía Patológica ${ }^{8}$. Este registro lleva funcionando desde 1993 y en él se recogen todos los diagnósticos de cáncer del hospital utilizando como fuentes de información el Servicio de Archivos y Codificación, el Archivo Central de Historias Clínicas y los archivos de pacientes de los servicios de anatomía patológica, radioterapia y oncología. Una vez obtenidos, estos registros se incorporan a una base de datos en la que se codifican los diagnósticos según la Clasificación Internacional de Enfermedades (CIE-9; CIE-10) ${ }^{9}$. El Centro 
Asistencial Universitario de León es el único hospital del área sanitaria y el Servicio Castellano y Leonés de Salud (Sacyl) tiene una cobertura cercana al $94 \%$, por lo que la gran mayoría de los casos de cáncer de mama del área de salud se diagnostican en él ${ }^{10}$.

En el estudio se incluyeron todos los diagnósticos registrados de neoplasia maligna de mama (CIE-9: 174, CIE-10: C50) desde 1 de enero de 1996 hasta el 31 de diciembre de 2010 , en mujeres con residencia en cualquier municipio del área de salud de León, según la tarjeta sanitaria. Al objeto de evitar duplicidades y evitar errores en el domicilio de residencia se comprobaron individualmente todos los casos seleccionados y se revisaron las coincidencias en fecha de nacimiento, número de historia o DNI. Así mismo se comprobó la coincidencia entre la residencia reflejada en la ficha de admisión con la de la tarjeta sanitaria.

Para el cálculo de las tasas se utilizó como denominador la población comunicada por el Instituto Nacional de Estadística por municipios, desagregada por sexo y edad (grupos quinquenales) ${ }^{11}$. En el año 1997 los datos no se ofrecían desagregados por sexo y edad, por lo que la población se calculó interpolando las cifras de las anualidades disponibles entre el padrón de 1996 y 1998. El área de salud de León está compuesta de 172 municipios. La población de mujeres varió de 185.000 mujeres al año de promedio en el primer trienio a 181.000 en el último. El 15\% tenía edad inferior a 20 años, el $40 \%$ entre 20 y 49 años y el $45 \%$ de la población femenina 50 años o más.

El programa de detección precoz de cáncer de mama de Castilla y León suministró información desagregada por año y grupo de edad de los casos detectados en el área de salud de León.

Análisis estadístico. Se calculó el número de casos detectados por cada mil mujeres en el cribado, desagregando en mujeres con menos de 50 años y en las que tenían 50 años y más así como el porcentaje de casos detectados durante el cribado respecto al total de casos registrados ${ }^{12}$.

Al objeto de reducir la variabilidad anual, se calcularon las tasas brutas y estandarizadas según la población mundial y europea por trienio (1996-98; 1999-01; 2002-04; 2005-07 y 2008-10) y las tasas estandarizadas según la población europea en las mujeres de 50 años y más y en las mujeres entre 20 y 49 años de edad.

Para el análisis de la distribución espacial de la incidencia se calcularon las razones estandarizadas de incidencias, tomando como tasas de referencia para el cálculo de los casos esperados las específicas para cada grupo de edad y sexo de todo el área de salud en el período. Para cada municipio se efectuó una estimación de los riesgos relativos (RR) municipales suavizados mediante el ajuste del modelo de Besag-York y Mollié ${ }^{13}$. Este modelo se ha extendido como la forma estándar para la elaboración de mapas de enfermedades, ya que resuelve de una forma muy eficiente el problema de las "áreas pequeñas". Se basa en el ajuste de modelos espaciales de Poisson con los casos observados como variable dependiente, los esperados como offset y dos términos de efectos aleatorios: 1) uno que es independiente para cada municipio y que representa la heterogeneidad municipal y 2) un término espacial que permite que cada municipio comparta su información promediándola con la de sus municipios vecinos. El criterio de vecindad empleado fue la adyacencia de contornos municipales. El modelo adopta la siguiente formulación:

$$
\text { Oi } \sim \operatorname{Po}\left(E_{i} \lambda_{i}\right) ; \log \left(\lambda_{i}\right)=\alpha+h_{i}+b_{i}
$$

donde: $\lambda_{\mathrm{i}}$ es el riesgo relativo en el área $\mathrm{i} ; \mathrm{O}_{\mathrm{i}}$ es el número de casos en el área $\mathrm{i}$; $\mathrm{E}_{\mathrm{i}}$ son los casos esperados; $\alpha$ es el intercepto; $h_{i}$ es el término de heterogeneidad municipal y $b_{i}$ el término espacial. 
Estos RR suavizados y sus probabilidades posteriores (PP) de que los RR fueran mayores de 1 se estimaron mediante métodos bayesianos ${ }^{14}$ empleando integrated nested Laplace approximation utilizando ( $\mathrm{R}$ INLA) ${ }^{15}$ con el programa $\mathrm{R}^{16}$.

Las PP de los RR $>1$ son el equivalente bayesiano del valor $\mathrm{p}$. Se consideró que los excesos de riesgo eran significativos cuando su probabilidad posterior fue igual o mayor de $0,8^{14}$.

\section{RESULTADOS}

El Registro de Tumores del Complejo Asistencial Universitario de León incluyó un total de 2.461 casos de cáncer de mama entre el 1 de enero de 1996 y el 31 de diciembre de 2010, de los que 2.379 correspondieron a mujeres residentes en municipios del área de salud de León. El 97,2\% de los casos registrados fueron diagnosticados por anatomía patológica y de éstos el 78,7\% correspondieron a carcinoma ductal infiltrante.

En la tabla 1 se puede observar la distribución de las tasas brutas y estandarizadas según la población mundial y europea en cada trienio. El número de casos diagnosticados se incrementó desde los 135 anuales de promedio en el trienio 1996-98 a 184 en el trienio 2008-10. Las tasas brutas ascendieron desde los 72,7 casos por 100.000 mujeres en el primer trienio hasta los 101,5 en el último. Las tasas estandarizadas se incrementaron en todos los trienios, en el caso de las tasas estandarizadas según la población mundial aumentaron de 43,2, en el primero a 51,4 casos nuevos por 100.000 mujeres en el último. Las tasas estandarizadas según la población europea pasaron de 58,0 a 69,4 casos nuevos por 10.000 mujeres, lo que supuso un incremento del 19,7\% en todo el período estudiado y un incremento anual promedio del 1,3\%.

En la tabla 2 se puede observar la distribución de los casos detectados distribuidos por edad, el número de mujeres incluidas en el cribado y los casos detectados en él. No se observó una tendencia clara en el número de casos diagnosticados en mujeres de menos de 50 años, manteniéndose durante el periodo estudiado en torno al $20 \%$ de los casos registrados.

El número de mujeres que participaron en el cribado se incrementó desde menos de 26.000 en el primer trienio a más de 41.000 en el último. En promedio se diagnosticaron 33 casos nuevos de cáncer de mama por cribado, de las que el $20 \%$ correspondieron a mujeres menores de 50 años de edad. El rendimiento del cribado fue superior a 5 por cada 1.000 mujeres en cribado en las mujeres mayores de 50 años en el primer trienio, estabilizándose en torno al 3 por 1.000 en los trienios sucesivos. En el caso de las mujeres de menos de 50 años el rendimiento se incrementó de 1,5 casos por cada 1.000 mujeres en cribado en el primer trienio a 2,2 en el

Tabla 1

Distribución de los casos, población y tasas de cáncer de mama femenino en el área de salud de León

\begin{tabular}{|c|c|c|c|c|c|}
\hline Trienio & Casos & Población & Tasa Bruta & \multicolumn{2}{|c|}{ Tasas Estandarizadas } \\
\hline & & & & Mundial & Europea \\
\hline $1996-98$ & 405 & 556870 & 72,7 & 43,2 & 58,0 \\
\hline $1999-01$ & 439 & 546105 & 80,4 & 44,8 & 60,0 \\
\hline $2002-04$ & 466 & 538532 & 86,5 & 46,6 & 62,6 \\
\hline $2005-07$ & 518 & 541735 & 95,6 & 50,9 & 68,1 \\
\hline $2008-10$ & 551 & 542918 & 101,5 & 51,4 & 69,4 \\
\hline
\end{tabular}


Tabla 2

Distribución de los casos detectados distribuidos por edad, el número de mujeres en cribado y los casos detectados por cribados

\begin{tabular}{|c|c|c|c|c|c|c|c|c|c|c|c|c|c|c|c|}
\hline \multirow{2}{*}{\begin{tabular}{|c} 
casos \\
/tasas
\end{tabular}} & \multicolumn{3}{|c|}{$\begin{array}{c}\text { casos registrados } \\
\text { (a) }\end{array}$} & \multicolumn{3}{|c|}{$\begin{array}{l}\text { mujeres en cribado } \\
\text { (b) }\end{array}$} & \multicolumn{3}{|c|}{$\begin{array}{l}\text { detectados } \\
\text { por cribado } \\
\text { (c) }\end{array}$} & \multicolumn{3}{|c|}{$(\mathrm{c} / \mathrm{b}) * 1000$} & \multicolumn{3}{|c|}{$(\mathrm{c} / \mathrm{a}) * 100$} \\
\hline & $\begin{array}{l}<50 \\
\text { años }\end{array}$ & $\begin{array}{l}>50 \\
\text { años }\end{array}$ & Total & $\begin{array}{l}<50 \\
\text { años }\end{array}$ & $\begin{array}{l}>50 \\
\text { años }\end{array}$ & Total & $\begin{array}{l}<50 \\
\text { años }\end{array}$ & $\begin{array}{l}>50 \\
\text { años }\end{array}$ & Total & $\begin{array}{l}<50 \\
\text { años }\end{array}$ & $\begin{array}{l}>50 \\
\text { años }\end{array}$ & Total & $\begin{array}{l}<50 \\
\text { años }\end{array}$ & $\begin{array}{l}>50 \\
\text { años }\end{array}$ & Total \\
\hline \multicolumn{16}{|l|}{ Trienio } \\
\hline $1996-98$ & 76 & 329 & 405 & 8128 & 17509 & 25637 & 12 & 98 & 110 & 1,48 & 5,60 & 4,29 & 15,8 & 29,8 & 27,2 \\
\hline 1999-01 & 93 & 346 & 439 & 8303 & 21311 & 29614 & 18 & 64 & 82 & 2,17 & 3,00 & 2,77 & 19,4 & 18,5 & 18,7 \\
\hline 2002-04 & 96 & 370 & 466 & 8413 & 21119 & 29532 & 19 & 58 & 77 & 2,26 & 2,75 & 2,61 & 19,8 & 15,7 & 16,5 \\
\hline $2005-07$ & 123 & 395 & 518 & 9395 & 27265 & 36660 & 27 & 79 & 106 & 2,87 & 2,90 & 2,89 & 22,0 & 20,0 & 20,5 \\
\hline $2008-10$ & 107 & 444 & 551 & 10621 & 30866 & 41487 & 23 & 98 & 121 & 2,17 & 3,18 & 2,92 & 21,5 & 22,1 & 22,0 \\
\hline
\end{tabular}

último trienio. Del mismo modo el porcentaje de casos detectados por cribado respecto al total de casos registrados descendió del $30 \%$ en las mujeres mayores de 50 años en el primer trienio al $22 \%$ en el último trienio y en el caso de mujeres menores de 50 años ascendió del $16 \%$ en el primer trienio al $22 \%$ en el último trienio.
En la figura 1 se puede observar la evolución de las tasas estandarizadas según la población europea en mujeres de 20 a 49 años y de 50 años o más. Se observa que en las mujeres de 20 a 49 años la incidencia ascendió desde 37,9 casos nuevos por 100.000 mujeres en el primer trienio (19961998) a 45,53 en el último (2008-2010) y en

Figura 1

Distribución de las tasas de incidencia de cáncer de mama en el área de salud de León por 100.000 mujeres

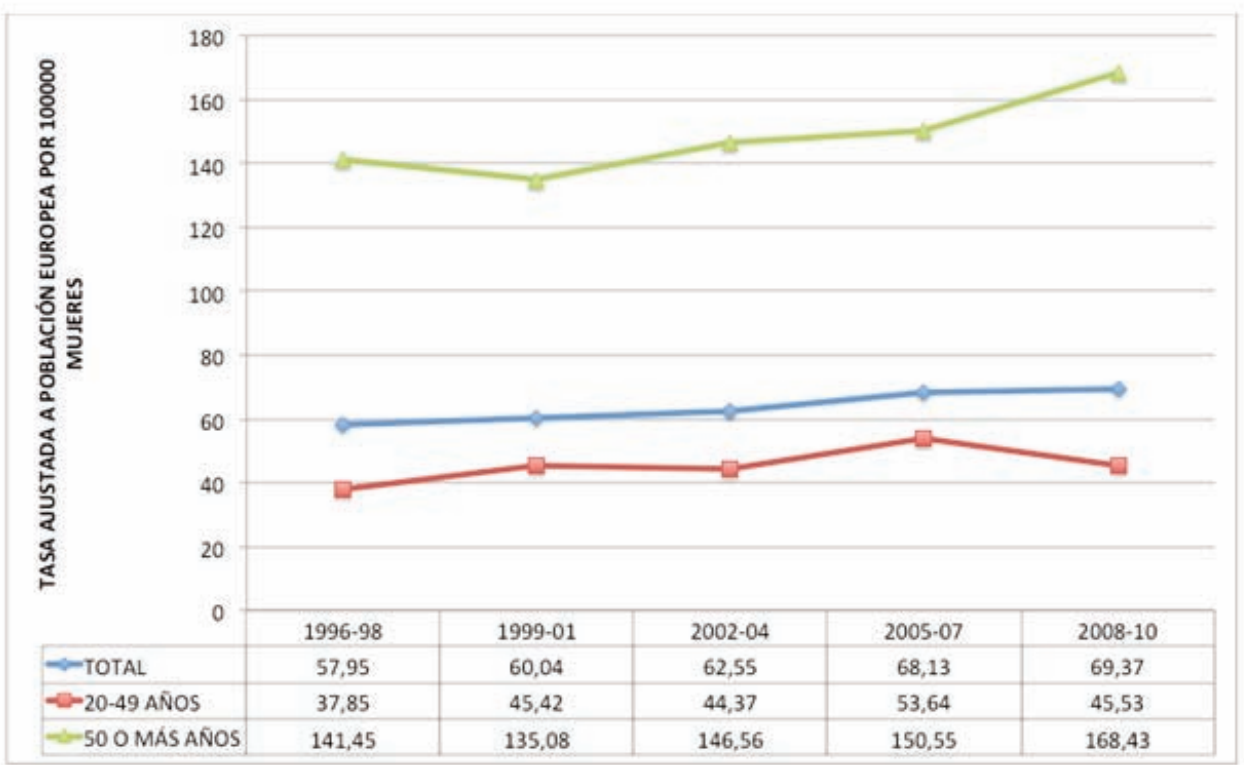




\section{Figura 2}

Distribución de las tasas de incidencia promedio según grupos de edad de cáncer de mama femenino en el área de salud de León (1994-2008)

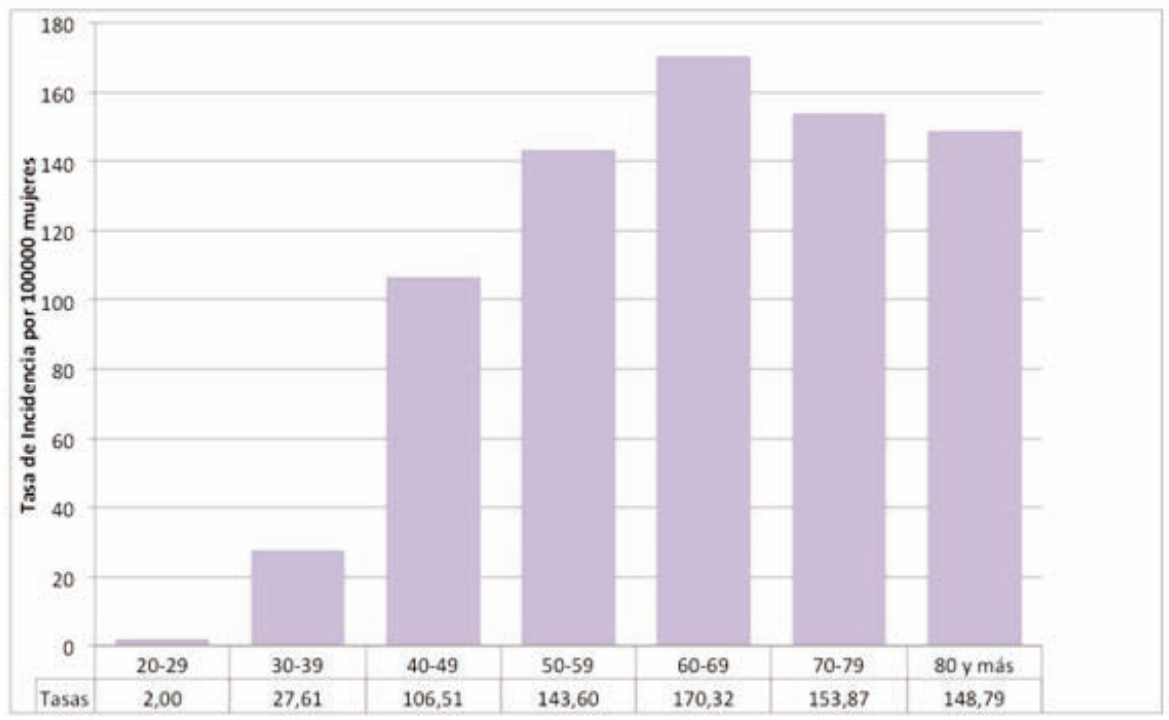

las mujeres de 50 años o más de 141,5 a 168,4 respectivamente, siendo en ambos casos el incremento observado entre el primer y último trienio en torno al $20 \%$.
En la figura 2 se puede observar como la incidencia de cáncer de mama se incrementó con la edad hasta el pico de máxima incidencia en el grupo de edad de 60-70 años en el que alcanzó los 170,3 casos por 100.000 mujeres en promedio.

\section{Figura 3}

Distribución geográfica de los riesgos relativos suavizados y de las probabilidades de que los riesgos relativos sean superiores a 1
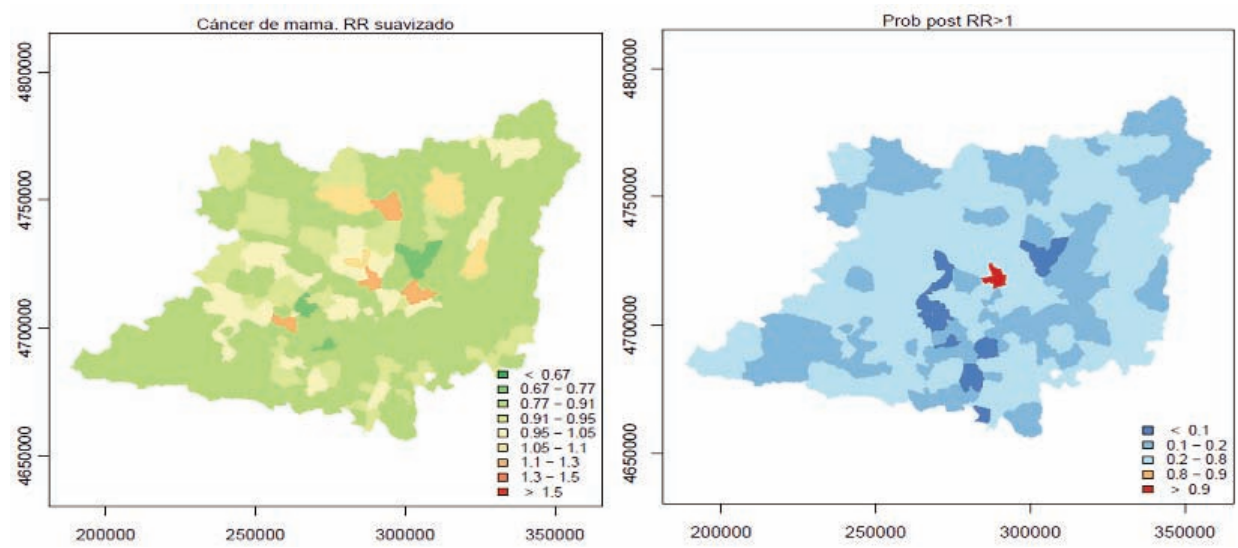
En la distribución geográfica (figura 3) ningún municipio presentó excesos de incidencia superiores al $20 \%$ y en cuatro fueron superiores al $10 \%$ (Villasabariego y Villarejo de Órbigo; RR=1,13 y León y Matallana de Torio $R R=1,11$ ). Las probabilidades a posteriori de que los RR fuesen superiores a 1 fueron superiores al 0,9 en el municipio de León y a 0,8 en Villarejo de Órbigo. Estas diferencias se explican por la distribución de la incidencia en el caso de las mujeres de 50 o más años en las que 7 municipios presentaban excesos de incidencia superiores al $10 \%$ e inferiores al $20 \%$ (Villarejo de Órbigo; RR=1,18; Villasabariego; $\mathrm{RR}=1,17$; Boñar y Matallana de Torío 1,16 y La Pola de Gordón, Astorga y León $\mathrm{RR}=1,11)$. En el caso de las mujeres de menos de 50 años no se observaron excesos de incidencia superiores al $10 \%$ en ningún municipio y en todos los casos la PP fue inferior a 0,8 .

\section{DISCUSIÓN}

En el área de salud de León, entre los años 1996 y 2010 se observa un incremento mantenido tanto del número de casos nuevos de carcinoma de mama, así como en las tasas brutas de incidencia, hasta superar los 180 casos nuevos anuales y una tasa bruta de 100 casos nuevos por 100.000 en el periodo 2008-2010. Esto es compatible con el envejecimiento observado en el área de salud, sin embargo, también la tasa estandarizada según población europea se incrementóanualmente. Este incremento es compatible con lo observado en los países desarrollados, lo cual se relaciona con el incremento del sedentarismo y la obesidad, así como con los cambios en la exposición hormonal: uso de anticonceptivos orales, menarquía temprana, menopausia tardía, nuliparidad y terapia hormonal sustitutoria $^{6}$. No obstante el incremento observado es inferior al estimado por Pollán et $\mathrm{al}^{7}$ para España, que se cifra en el 3\% de incremento anual. El incremento obser- vado en el ASL ha sido similar en las mujeres de menos de 50 años y en las de 50 ó más, en torno al $20 \%$ en los 15 años estudiados.

La tasa de incidencia estandarizada según la población mundial observada en el ASL estuvo en torno a 50 casos por 100.000 mujeres, muy por debajo de lo observado en la mayoría de los países desarrollados: en torno a los 90 casos nuevos por 100.000 mujeres ${ }^{2}$. Lo mismo observamos con relación a las tasas estimadas para la UE-27, con tasas ajustadas según la población europea cercanas a 110 casos nuevos por 100.000 mujeres en comparación con tasas inferiores a 70 casos nuevos por 100.000 mujeres observadas en el área de salud de León ${ }^{3}$. En comparación con España, donde se estima en cerca de 85 casos nuevos por 100.000 mujeres, también las tasas observadas en el ASL son inferiores. Según los registros españoles, tan solo Albacete y Cuenca presentan tasas inferiores a las nuestras ${ }^{17}$.

En Castilla y León el programa de cribado de cáncer de mama mediante mamografía se inició en el año 1992 y por lo tanto llevaba cuatro años de funcionamiento cuando se inició nuestro estudio. En el caso de ASL el programa se detectó un menor número de mujeres respecto al promedio de programas de cribado españoles que están en crifras superiores a 3,5 casos nuevos por 1.000 mujeres estudiadas e inferiores también a los programas de detección precoz de cáncer de mama europeos, donde se diagnostican 4 casos por cada 1.000 mujeres cribadas de 50 a 69 años ${ }^{18}$. Estas diferencias pueden ser explicadas por las menores tasas de incidencia del ASL.

Con relación a la distribución municipal de la incidencia en el área de salud de León observamos que ningún municipio presentó riesgos superiores al 20\% y cómo la distribución geográfica fue muy 
"plana". Solo en el caso de un municipio se observó una $\mathrm{PP}>0,9$, es decir, sólo en dicho municipio se tienen una certeza superior al $90 \%$ de que el riesgo es significativamente superior a la media del territorio. Esto coincide con lo observado en los Atlas de Mortalidad donde las diferencias entre municipios y/o áreas censales no son muy elevadas ${ }^{19,20}$ lo que de alguna manera puede ser explicado por las pequeñas variaciones en la exposición a los factores hormonales y reproductivos que determinan la incidencia del cáncer de mama.

La principal limitación del estudio es que la fuente de los casos es un registro hospitalario y no un registro poblacional. Aunque el objetivo y la finalidad principal de un registro hospitalario es suministrar información para evaluar la calidad de la asistencia a los pacientes diagnosticados de cáncer y/o atendidos en el hospital, son también de utilidad para conocer la frecuencia anual de casos y su tendencia en función de las diferentes características de los pacientes $^{8}$. Habitualmente subestiman el número de casos, en mayor o menor medida, en función del nivel de cobertura del hospital y del acceso de la población de referencia. Ello viene dado por diversos factores: el número de hospitales en el área y el grado de cobertura del servicio autonómico de salud. El Complejo Asistencial Universitario de León es el único hospital del área y el Servicio Castellano y Leonés de Salud (Sacyl) y tiene una cobertura cercana al 94\%. Además, el servicio de anatomía patológica diagnostica incluso a ciudadanos que tienen coberturas privadas o mutuales diferentes del Sacyl. Todo ello nos lleva a pensar que son pocos los cánceres de mama que no son diagnosticados o atendidos en el mismo. En cualquier caso, las tasas observadas sí nos pueden dar una idea de la incidencia mínima del área y de la tendencia temporal de la misma.

A modo de conclusión se puede decir que las tasas de incidencia de cáncer de mama observadas en el área de salud de León son de las más bajas de nuestro país y aunque se ha observado una tendencia creciente en los últi- mos años es inferior a la observada en otros lugares. No se observan diferencias relevantes en la distribución geográfica. A pesar de todo ello se diagnostican más de 180 nuevos casos al año, lo que obliga a prestar una especial atención a la detección precoz y a la promoción de hábitos de vida saludables.

\section{BIBLIOGRAFÍA}

1. Ferlay J, Shin HR, Bray F, Forman D, Mathers C, Parkin DM. Estimates of worldwide burden of cancer in 2008: GLOBOCAN 2008. Int J Cancer. 2010; 127(12):2893-917.

2. Jemal A, Bray F, Center MM, Ferlay J, Ward E, Forman D. Global cancer statistics. CA Cancer J Clin. 2011;61:6990 .

3. Ferlay J, Steliarova-Foucher E, Lortet-Tieulent J, Rosso S, Coebergh JW, Comber H, et al. Cancer incidence and mortality patterns in Europe: estimates for 40 countries in 2012. Eur J Cancer. 2013; 49(6):1374-403.

4. Hankinson SE, Colditz GA, Willett WC (2004) Towards an integrated model for breast cancer etiology: the lifelong interplay of genes, lifestyle, and hormones. Breast Cancer Res. 6(5):213-218.

5. Pollán M. Epidemiology of breast cancer in young women. Breast Cancer Res Treat. 2010; Suppl 1:3-6.

6. Pollán M, Michelena MJ, Ardanaz E, Izquierdo A, Sánchez-Pérez MJ, Torrella A. Breast cancer incidence in Spain before, during and after the implementation of screening programmes. Ann Oncol. 2010; 21 (Supplement 3): iii97-iii102.

7. Pollán M, Ramis R, Aragonés N, Pérez-Gómez B, Gómez D, Lope V. Municipal distribution of breast cancer mortality among women in Spain. BMC Cancer. 2007;7:78.

8. Grupo Nacional Multidisciplinar de Registros Hospitalarios de Tumores. Manual de procedimientos en los Registros Hospitalarios de Tumores. Málaga: Sociedad Española de Anatomía Patológica; 2007.

9. 16. Clasificación Internacional de Enfermedades. 9a revisión. Modificación clínica, 7a ed. eCIE9MC. Edición electrónica de la CIE-9-MC. Madrid: Ministerio de Sanidad y Política social; 2011.

10. Naveiro JC, Peral A, Díez A, González FA, Burón JL. Incidencia y riesgo de cáncer en el área sanitaria de León. Oncología. 2001; 24:21-28. 
11. Instituto Nacional de Estadística. INEbase, población por municipios desagregada por sexo y edad (grupos quinquenales). Disponible en: http://www.ine.es

12. Programa de detección precoz de cáncer de mama de Castilla y León. Valladolid: Junta de Castilla y León; 2005.

13. Besag J, York J, Mollié A. Bayesian image restoration, with two applications in spatial statistics. Ann Inst Stat Math. 1991; 43:1-59.

14. Richardson S, Thomson A, Best N, Elliott P. Interpreting posterior relative risk estimates in diseasemapping studies. Environ Health Perspect. 2004; 112: 1016-25.

15. Rue H, Martino S, Chopin N. Approximate Bayesian Inference for Latent Gaussian Models Using Integrated Nested Laplace Approximations (with discussion). J Royal Statistic Soc Series B. 2009; 61: 319392.

16. The R Development Core Team. R: A language and environment for statistical computing. Vienna: R Foundation for Statistical Computing; 2010. Disponible en: http://www.R-project.org/

17. Centro Nacional de Epidemiología. La situación del cáncer en España, 1975-2006. Madrid: Instituto de Salud Carlos III; 2009.

18. González A, González MJ. Los programas de detección precoz del cáncer de mama en España. Psicooncología. 2007; 4 (2-3):249-263.

19. Lopez-Abente G, Ramis R, Pollán M, et al. Atlas municipal de mortalidad por cáncer en España 19891998. Madrid: Instituto de Salud Carlos III; 2006.

20. De Rovira JB, Martínez JM. Atlas de mortalidad en municipios y unidades censales de España 1984-2004. Fundación BBVA; 2013. 